\title{
Increased mean platelet volume in patients with vestibular migraine
}

\author{
Mehmet Emin Çavuş ${ }^{1}$, Talip Talha Tanyeli ${ }^{2} \oplus$, Fatih Alper Akcan $^{3} \oplus$, Ömer Bayır ${ }^{1}$, Didem \\ Gökkurt ${ }^{4} \oplus$
}

\begin{abstract}
${ }^{1}$ Department of Otorhinolaryngology, University of Health Sciences, Dışkapı Yıldırım Beyazıt Training and Research Hospital, Ankara, Turkey

${ }^{2}$ Department of Otorhinolaryngology, Ministry of Health Polatl State Hospital, Ankara, Turkey

${ }^{3}$ Department of Otorhinolaryngology, Düzce University School of Medicine, Düzce, Turkey

${ }^{4}$ Department of Neurology, Ministry of Health Polatl State Hospital, Ankara, Turkey
\end{abstract}

\section{ABSTRACT}

Objectives: The purpose of this study was to investigate relation between mean platelet volume, platelet distribution width, platelet-to-lymphocyte ratio, neutrophil-to-lymphocyte ratio and vestibular migraine.

Methods: This study was planned in prospective manner and conducted in Ankara Polatli State Hospital between April 2017 and May 2018. Thirty patients diagnosed with vestibular migraine and thirty healthy, age and sex-matched subjects were enrolled to the study. Mean platelet volume, platelet distribution width, platelet count, neutrophil count and lymphocyte count in blood samples were measured.

Results: The mean age of the patients with vestibular migraine was $39.90 \pm 7.16$ and the study group consisted of 11 males and 19 females. The mean platelet volume and platelet distribution width values in patients with vestibular migraine were significantly higher than the control group $(p<0.001)$. There were no difference on mean neutrophil/lymphocyte ratio and platelet/lymphocyte ratio values between patient group and control group.

Conclusions: We found a positive relation of increased levels of mean platelet volume and platelet distribution width with vestibular migraine. Higher mean platelet volume is associated with atherosclerosis and thromboembolism. Vascular pathologies are blamed for both headache syndromes like migraine and vertiginous syndromes. This theory supports our study but pathophysiological mechanism is not clear.

Keywords: Hematologic parameters, mean platelet volume, neutrophil-to-lymphocyte ratio, platelet-tolymphocyte ratio, vestibular migraine

$\mathrm{V}$ estibular migraine is an important clinical syndrome characterised by migraine type headaches accompanied with vertigo. Relation between migraine and vertigo is a complex issue and not well understood. Migraine and vertigo are common problems and a patient may have both of them by chance [1]. Thirty eight percent of migraine patients have vertigo [2-3].
Also many vertiginous pathologies are associated with migraine epidemiologically. Benign paroxysmal positional vertigo, Meniere disease, motion sickness and cerebellar disorders are associated with migraine [1]. So differentiation of vestibular migraine from these pathologies is a clinical challenge. Vestibular migraine is one of the frequent causes of both headache and ver- 
tigo. Migraine prevalence is $16 \%$ and vertigo prevalence is $7 \%$ [1]. Acoording to these values expected comorbidity of vertigo and migraine is $1.1 \%$ but Neuhauser et al. [4] showed vestibular migraine prevalence as $3.2 \%$. In the view of such information many authors think vestibular migraine as a spesific clinical entity rather than coincidental coexistence of vertigo and migraine.

Diagnosis of vestibular migraine is a challenge because pathophysiology is not well understood and there is no specific examination finding, biological marker or laboratory test [2]. Detailed and careful history is important and high index of suspicion is needed for clinician. Because of difficulties abuot diagnosis important part of patients are misdiagnosed [3].

Mean platelet volume (MPV) is average size of platelets and included in complete blood count. MPV gives information about platelet production rate. If platelet production accelerates in bone marrow MPV gets higher. Enlarged platelets have higher activity and produce vasoactive substances, so high MPV levels are associted with vasospasm and thrombosis [5]. Increased levels of MPV, Platelet Distribution Width (PDW), Platelet-to-Lymphocyte Ratio (PLR) and Neutrophil-to-Lymphocyte Ratio (NLR) are also associated with inflammatory pathologies and vascular pathologies such as atherosclerosis, thromboembolism [6].

There are many theories for explanation of pathophysiology of vestibular migraine. One of these theories is reversible vasospasm of the internal auditory artery or its branches. This vasospasm causes the ischemia of the labyrinth and then resulting in cochleovestibular dysfunction and vertigo experienced in vestibular migraine [7-9].

MPV, PDW, PLR and NLR values in vestibular migraine have not been investigated yet and we aimed to show if there is a relation of these parameters with vestibular migraine.

\section{METHODS}

This study was approved by the local ethics committee for non-invasive research and conforms to the principles of the Declaration of Helsinki. Written informed consent was obtained from all patients at the beginning of the study.

This study was planned in prospective manner and conducted in Ankara Polatl State Hospital between April 2017 and May 2018. Thirty patients diagnosed with definite vestibular migraine according to the modified Neuhauser criteria by Lempert et al. [10] (Table 1) and thirty healthy, age and sex-matched subjects were enrolled to the study. Patients were diagnosed with vestibular migraine by neurology specialist and referred to otolaryngology clinic. All patients underwent a detailed otolaryngologic and neurologic examination. Brain and ear magnetic resonance imaging with using gadolinium as a contrast medium were obtained from all patients.

Patients were excluded from the study if they had

\section{Table 1. Vestibular migraine diagnostic criteria [10]}

A. At least 5 episodes with vestibular symptoms of moderate or severe intensity, lasting 5 minutes to 72 hours

B. Current or previous history of migraine with or without aura

C. One or more migraine features with at least $50 \%$ of the vestibular episodes

1. headache with at least two of the following four characteristics:
a) one sided location
b) pulsating quality
c) moderate or severe pain intensity
d) aggravation by routine physical activity
2. photophobia and phonophobia
3. visual aura

D. Not better accounted for by another vestibular or ICHD diagnosis 
acute inflammation, infection, pneumonia, chronic obstructive pulmonary disease (COPD), asthma, the diagnosis of acute or chronic pulmonary thromboembolism, coronary artery disease, congestive heart failure, renal and hepatic dysfunction, hematological diseases, cancer, auto-immune diseases, diabetes mellitus, systemic hypertension, obstructive sleep apnea, connective tissue diseases, inflammatory bowel diseases, the use of antithrombotic agents, and smoking history.

\section{Biochemical analyses}

MPV, PDW, platelet count, neutrophil count and lymphocyte count in blood samples were measured. Venous blood samples were obtained from antecubital vein at the time of vertigo attack and collected into tubes containing ethylenediaminetetraacetic acid (EDTA) at 9 am. following an overnight fast. To avoid platelet swelling measurements were done shorter than 30 minutes after sampling. An automated blood cell counter was used for these measurements (Horiba ABX Pentra DX 120).

\section{Statistical Analysis}

Continuous data were summarized as mean \pm standard deviation, and categorical data were as frequency and percentage. Independent sample $t$ test was used to compare groups for continuos variables. Relation between categorical variables were analyzed by Chi-Square test. Statistical analyses were done with SPSS v.22.0 for Windows statistical package, and statistical significance level was considered as 0.05 .

\section{RESULTS}

The mean age of the patients with vestibular migraine were $39.90 \pm 7.16$ years and $37.93 \pm 7.62$ years for the control group. The study group consisted of 11 males and 19 females and the control group consisted of 17 males and 13 females. There was no significant difference between age or gender in two $p$ $=0.307$ and $p=0.121$, respectively) (Table 2).

The mean MPV values were $9.93 \pm 0.68 \mathrm{fl}$ in vestibular migraine patients and $8.24 \pm 0.75 \mathrm{fl}$ in the control group. The mean MPV values in patients with vestibular migraine were significantly higher than the

Table 2. Demographic data of the study and the control groups

\begin{tabular}{lccc}
\hline & Study group & $\begin{array}{c}\text { Control } \\
\text { group }\end{array}$ & $\boldsymbol{p}$ value \\
\hline Age & $39.90 \pm 7.16$ & $37.93 \pm 7.62$ & 0.307 \\
Sex & & & \\
Female & $19(63.3 \%)$ & $13(43.3 \%)$ & 0.121 \\
Male & $11(36.7 \%)$ & $17(56.7 \%)$ & \\
\hline
\end{tabular}

The data are expressed in mean \pm standard deviation or in numbers and percentages.

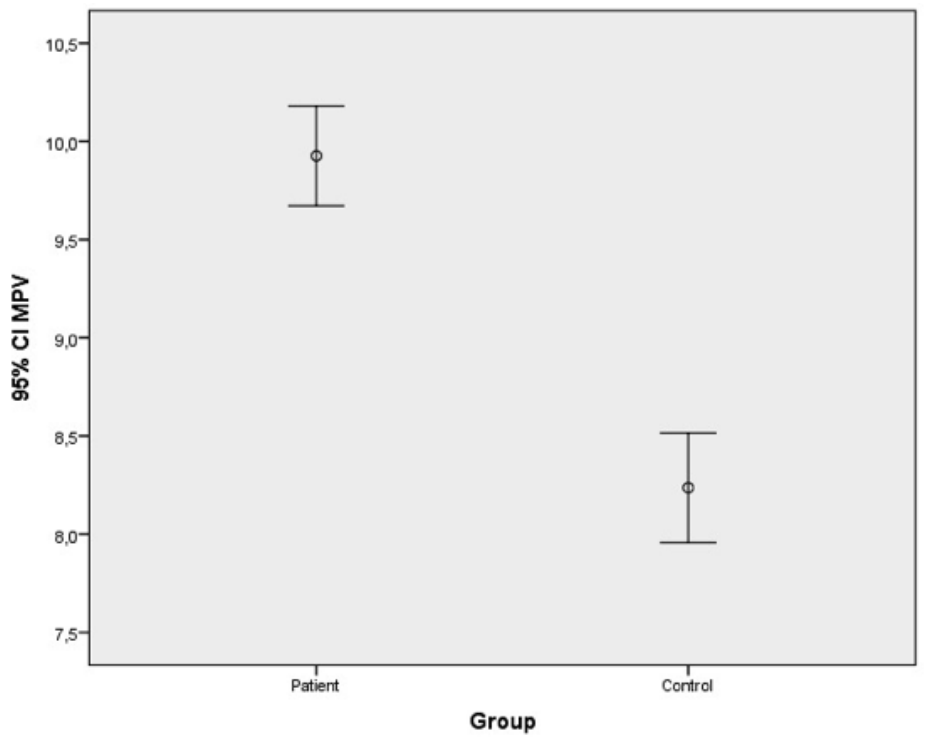

Fig. 1. The mean MPV values of the patients with migrainous vertigo and the control group. 
Table 3. Comparison of themean MPV, PDW values and platelet counts of the study and the control groups

\begin{tabular}{lccc}
\hline & Study group & Control group & $\boldsymbol{p}$ value \\
\hline MPV (fl) & $9.93 \pm 0.68$ & $8.24 \pm 0.75$ & $<\mathbf{0 . 0 0 1 *}$ \\
PDW (\%) & $18.67 \pm 1.53$ & $14.08 \pm 2.06$ & $<\mathbf{0 . 0 0 1 *}$ \\
Plateletcount $\left(10^{3} / \mathrm{mm}^{3}\right)$ & $274.83 \pm 64.58$ & $306.00 \pm 106.32$ & 0.175 \\
\hline
\end{tabular}

The data are expressed in mean \pm standard deviation. MPV $=$ mean platelet volume, $\mathrm{PDW}=$ platelet distribution width.

${ }^{*} p<0.05$ value was regarded as significant while the significant differences between the groups are shown in bold.

control group $(p<0.001)$ (Fig. 1). The mean PDW values were $18.67 \% \pm 1.53 \%$ in vestibular migraine patients and $14.08 \% \pm 2.06 \%$ in control group. The mean PDW values in patients with vestibular migraine were significantly higher than the controls $(p<0.001)$. The mean values of platelet count of the patients and the control group were $274.83 \pm 64.58(103 / \mathrm{mm} 3)$ and
$306.00 \pm 106.32(103 / \mathrm{mm} 3)$, respectively. There were no difference on platelet count levels between groups $(p=0.175)$ (Table 3).

The mean NLR values were $2.19 \pm 0.86$ in the patient group and $2.15 \pm 1.52$ in the control group. The mean PLR values were $138.24 \pm 50.83$ in the patient group and $127.39 \pm 45.31$ in the control group. There

Table 4. Comparison of themean NLR and PLR ratiovalues of the study and the control groups

\begin{tabular}{lccc}
\hline & Study group & Control group & $\boldsymbol{p}$ value \\
\hline NLR & $2.19 \pm 0.86$ & $2.15 \pm 1.52$ & 0.902 \\
PLR & $138.24 \pm 50.83$ & $127.39 \pm 45.31$ & 0.387 \\
\hline
\end{tabular}

The data are expressed in mean \pm standard deviation. NLR $=$ neutrophil-to-lymphocyte ratio, PLR = platelet-tolymphocyte ratio

were no difference on NLR and PLR between groups ( $p=0.902$ and $p=0.387$, respectively) (Table 4).

\section{DISCUSSION}

Pathogenesis of vestibular migraine is not well understood but there are various etiopathogenetic theories. According to cortical spreading theory cortical depolarisation wave produce vertigo [8]. Some neuropeptides are released asymmetricly and influence vestibular centers. This neuropeptid stimulation may cause vertigo at the time of migraine attack [11]. Another explanation is based on internal auditory artery spasm - may also be caused by serotonin- at the time migraine attack [7-9].

MPV and platelet count are calculated in complete blood count test routinely and MPV is unnoticed by the clinicians most of the time. MPV and platelet count levels give information about vasospasm and thrombotic function [6]. Main finding of our study was that MPV levels were significantly higher in vestibular migrainepatients than the control group.

Relation of MPV and migraine or other headache syndromes is not investigated yet and there is only single study in literature which investigated possible relation of MPV and vertigo. Celikbilek et al. [12] showed MPV levels significantly higher in benign paroxysmal vertigo patients than in controls.

Some authors studied MPV values in patients with inner ear diseases and showed controversial results. Kemal et al. [13] and Sarıkaya et al. [14] reported that MPV values were significantly higher in tinnitus patients than the control group. But Beyan and Beyan [15] reported that there was no relation between high levels of MPV values with tinnitus. Recent study by 
Ulusoy et al. [16] showed that MPV values were significantly higher in tinnitus patients than the control group. MPV values were studied also in sudden sensorineural hearing loss (SSHL) patients and results were controversial like tinnitus patients. Ulu et al. [17] and Sagit et al. [18] found high levels of MPV were associated with SSHL significantly. Durmus et al. [19] also reported that MPV values were significantly higher in SSHL patients than the control group [19]. In other two studies by Karli et al. [20] and Kum et al. [21] mean MPV values of SSHL patients were higher than the control grup but differences were not significant. Ozturk et al. [22] also found no difference between SSHL and control group MPV values.

This is the first study which investigates relation of mean platelet volume with vestibular migraine. In our study we found a positive relation of increased levels of MPV with vestibular migraine. But mechanism of this positive relation is not clear. We know that higher MPV is associted with vasospasm and thromboembolism [23]. Vascular pathologies are blamed for both headache syndromes like migraine and vertiginous syndromes. This theory supports our study but pathophysiological mechanism is not understood yet.

\section{CONCLUSION}

MPV can be considered as a practical and valuable parameter for assessment of vestibular migraine. And further studies are needed for explanation of relation between MPV values and vestibular migraine.

\section{Conflict of interest}

The authors disclosed no conflict of interest during the preparation or publication of this manuscript.

\section{Financing}

The authors disclosed that they did not receive any grant during conduction or writing of this study.

\section{REFERENCES}

1. Lempert T, Neuhauser HK. Epidemiology of vertigo, migraine and vestibular migraine. J Neurol 2009;256:333-8.

2. Baker BJ, Curtis A, Trueblood P, Vanqsnes E. Vestibular functioning and migraine: comparing those with and without vertigo to a normal population. J Laryngol Otol 2013;127:116976.

3. Von Brevern A, Radtke A, Clarke AH, Lempert T. Migrainous vertigo presenting as episodic positional vertigo. Neurology 2004;62:469-72.

4. Neuhauser HK, Radtke A, Von Brevern A, Feldmann M, Lezius F, Ziese T, et al. Migrainous vertigo: prevalence and impact on quality of life. Neurology 2006;67:1028-33.

5. Briggs C. Quality counts: new parameters in blood cell counting. Int Jnl Lab Hem 2009;31:277-97.

6. Machin SJ, Briggs C. Mean platelet volume: a quick easy determinant of thrombotic risk? J Thromb and Haemost 2009;8:146-7.

7. Baloh RW. Neurotology of migraine. Headache 1997;37:61521.

8. Felisati G, Pipolo C, Portaleone S. Migraine and vertigo: two diseases with the same pathogenesis? Neurol Sci 2010;31:1079.

9. Killer HE, Forrer A, Flammer J. Retinal vasospasm during an attack of migraine. Retina 2003;23:253-4.

10. Lempert T, Olesen J, Furman J, Waterston J, Seemungal B, Carey J, et al. Vestibular migraine: diagnostic criteria. J Vestib Res 2012;22:167-72.

11. Eggers SD. Migraine-related vertigo: diagnosis and treatment. Curr Pain Headache Rep 2007;11:217-26.

12. Celikbilek A, Tanik N, Zarasiz G, Celikbilek M. Do platelet indices have a role in benign paroxysmal positional vertigo? Neurol Res 2014;36:763-8.

13. Kemal O, Muderris T, Basar F, Kutlar G, Gül F. Prognostic value of mean platelet volume on tinnitus. J Laryngol Oto 2016;130:162-5.

14. Sarıkaya Y, Bayraktar C, Karatas M, Dogan S, Olt S, Kaskalan E, et al. Increased mean platelet volume in patients with idiopathic subjective tinnitus. Eur Arch Otorhinolaryngol 2016;273:3533-6.

15. Beyan C, Beyan E. Mean platelet volume may not be increased in patients with idiopathic subjective tinnitus. Eur Arch Otorhinolaryngol 2016;273:4035-6.

16. Ulusoy B, Bozdemir K, Akyol M, Mise HI, Kutluhan A, Korkmaz MH. Investigation of neutrophil-to-lymphocyte ratio, platelet-to-lymphocyte ratio and mean platelet volume in patients with tinnitus. J Laryngol Otol 2018;132:129-32.

17. Ulu S, Ulu MS, Ahsen A, Yucedag F, Aycicek A, Celik S. Increased levels of mean platelet volume: a possible relationship with idiopathic sudden hearing loss. Eur Arch Otorhinolaryngol 2013;270:2875-8.

18. Sagit M, Kavugudurmaz M, Guler S, Somdas MA. Impact of mean platelet volume on the occurrence and severity of sudden sensorineural hearing loss. J Laryngol Otol 2013;127:972-6.

19. Durmus K, Terzi H, Karatas TD, Dogan M, Uysal IO, Sencan $\mathrm{M}$, et al. Assessment of hematological factors involved in development and prognosis of idiopathic sudden sensorineural hearing loss. J Craniofacial Surg 2016;27:85-91.

20. Karli R, Alacam H, Unal R, Kucuk H, Aksoy A, Ayhan E. Mean platelet volume: is it a predictive parameter in the diagnosisof sudden sensorineural hearing loss? Indian J 
Otolaryngol Head Neck Surg 2013;65:350-3.

21. Kum RO, Özcan M, Baklacı D, Yurtsever Kum N, Yilmaz YF, Unal A, et al. Investigation of neutrophil-to-lymphocyte ratio and mean platelet volume in sudden hearing loss. Braz J Otorhinolaryngol 2015;81:636-41.

22. Ozturk M, Kara A, Dasli S, Topdag M. Mean platelet volume: is it a parameter associated with idiopathic sensorineural hearing loss? Eur Arch Otorhinolaryngol 2014;271:2595-6.

23. Capaccio P, Ottaviani F, Cuccarini V, Bottero A, Schindler A, Cesana BM, et al. Genetic and acquired prothrombotic risk factors and sudden hearing loss. Laryngoscope 2007;117:547-51. 